\section{Dyspnoea perception and susceptibility to exacerbation in COPD}

\author{
Heidi A Ridsdale, 1,2 John R Hurst ${ }^{2,3,4}$
}

COPD is a heterogeneous condition. This concept has evolved from classic descriptions of pink puffers and blue bloaters to a more complex understanding of disease phenotypes. Phenotypes are relevant because they may predict an important clinical outcome or a specific underlying disease mechanism (endotype), either or both of which might suggest benefit from a particular intervention-'personalised medicine'. In addition to the progressive decline in lung function, symptoms and physical performance that characterise COPD, (some) patients are prone to periodic deteriorations in respiratory health called exacerbations. And given that exacerbations are responsible for much of the morbidity, mortality and therefore healthcare costs associated with COPD, exacerbation-susceptible patients or 'frequent exacerbators' form a clinically relevant phenotype. Why does one patient get more exacerbations than another? A simple clinical question, without a simple answer.

To this first level of complexity in COPD-phenotypes-we must add that exacerbations themselves are heterogeneous events. Assume, for a moment, that a patient is truly having an exacerbation (and not one of the other pathologies that can cause symptom deterioration in $\mathrm{COPD}^{1}$ ). That exacerbation might be caused by acquisition of a respiratory virus, alterations in the airway bacterial microbiome, both or indeed neither and these are associated with different patterns of symptoms and inflammation. ${ }^{2}$ Thus, if we are going to prevent and mitigate exacerbations, we need to ask first why is this patient a frequent exacerbator and then what type of exacerbations is this frequent exacerbator susceptible to? Do not be fooled in to thinking that prevention of exacerbations in COPD is easy.

Some patients appear durably more susceptible to exacerbations than others, the 'frequent exacerbator' phenotype, ${ }^{3}$ and

\footnotetext{
${ }^{1}$ Central and North West London NHS Foundation Trust, London, UK; ${ }^{2}$ The Camden COPD Service, ${ }^{3} \mathrm{UCL}$ Respiratory, UCL Medical School, University College London, London, UK; ${ }^{4}$ Royal Free London NHS Foundation Trust, London, UK
}

Correspondence to Dr John R Hurst, UCL Respiratory, UCL Medical School, University College London, London NW3 2QG, UK; j.hurst@ucl.ac.uk the associations of exacerbation frequency in COPD—note associations, not causes are well described. They include disease severity, female sex, and the presence of gastro-oesophageal reflux, all of which increase the risk of exacerbation. Specific mechanisms to explain exacerbations have been harder to define-and thus the concept that exacerbation susceptibility is an endotype remains more challenging. Some studies have examined various functions of the immune system, for example, ${ }^{4}$ a plausible target, but what if the explanation is something much more clinical? What if symptom perception differs in those susceptible to frequent exacerbations? All those engaged with exercise interventions in COPD will recognise that the perception of dyspnoea for a given task varies greatly between patients. ${ }^{5}$ Do frequent exacerbators simply have a greater perception of breathlessness? A paper in Thorax sheds light on this area. ${ }^{6}$

Scioscia et $a l^{6}$ report a small experimental medicine study in which the perception of breathlessness and ventilatory response to hypercapnoea were assessed in 34 patients with stable COPD (14 frequent and 20 infrequent exacerbators) and 10 healthy controls. The bottom line is that there was no statistically significant difference in the ventilatory response by exacerbation susceptibility status (though this was diminished in COPD patients compared with controls), but there was a difference in breathlessness perception. Dyspnoea perception was greater in frequent exacerbators compared with infrequent, and, oddly, the infrequent exacerbators had a blunted response compared with the controls. This is a difficult work to do and we applaud the investigators for their effort, but the study is not without flaws. Notably, there were clinically relevant differences in disease severity between the COPD groups that did not reach statistical significance because of the study size (eg, $50 \%$ vs $21 \%$ GOLD Stage 2). Such differences remain an explanation for the reported alterations in dyspnoea perception. Also, patients with respiratory failure and the sorts of cardiac comorbidities that are important in clinical practice were excluded.

So would reducing dyspnoea perception be a good way to mitigate exacerbations of COPD? Perhaps. But there is the more troubling concern that perception of breathlessness in the context of an exacerbation could be a good thing. Not perceiving an exacerbation, and therefore not treating it, could result in greater lung damage. We know, for example, that a greater proportion of unreported (untreated) exacerbations can be associated with poorer health outcomes including greater risk of hospitalisation. ${ }^{7}$ However, in the latter study, unreported exacerbations had been perceived as changes in symptoms, they just had not presented for therapy. Could there be a group of subclinical exacerbations that also contribute to progression of lung disease and which are at present ignored?

Like all good research, this paper raises more questions than it answers. What can we learn? First, we should be noting for an individual patient how breathless they get with exercise (routinely measured as part of pulmonary rehabilitation) and asking whether results suggest that the patient has altered perception. Because if they do, then intervention to affect that-learned breathing techniques for example-just might help and may be more likely to help than, say, inhaled corticosteroids. Better yet, why not an adequately powered trial of such an intervention in a group of patients with altered dyspnoea perception? Meanwhile, in the same way that not every patient with COPD is the same, not every frequent exacerbator is the same, and it is likely that not every frequent exacerbator gets exacerbations for the same reasons. Remember this in your next clinic: right intervention, right patient, right time.

Correction notice This article has been corrected since it was published Online First. In the second paragraph, the word 'hypercapnoea' has been corrected to 'heterogeneous'. In the fourth paragraph, the word 'hypercapnia' has been corrected to 'hypercapnoea'.

Contributors HAR and JRH developed the concept. JRH wrote the first draft. HAR and JRH reviewed the draft and agreed the final version.

\section{Competing interests None.}

Provenance and peer review Commissioned; externally peer reviewed.

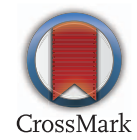

To cite Ridsdale HA, Hurst JR. Thorax 2017;72:107108.

Published Online First 4 November 2016

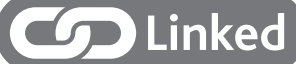

- http://dx.doi.org/10.1136/thoraxjnl-2016-208332 


\section{Editorial}

Thorax 2017;72:107-108.

doi:10.1136/thoraxjnl-2016-209318

\section{REFERENCES}

1 Hurst JR, Wedzicha JA. What is (and what is not) an exacerbation of COPD: thoughts from the new GOLD guidelines. Thorax 2007:62:198-9.

2 Bafadhel M, McKenna S, Terry S, et al. Acute exacerbations of chronic obstructive pulmonary disease: identification of biologic clusters and their biomarkers. Am J Respir Crit Care Med 2011;18:662-71.

3 Hurst JR, Vestbo J, Anzueto A, et al. Susceptibility to exacerbation in chronic obstructive pulmonary disease. N Engl I Med 2010;363:1128-38.

4 Berenson CS, Kruzel RL, Eberhardt E, et al. Impaired innate immune alveolar macrophage response and the predilection for COPD exacerbations. Thorax 2014;69:81181-8.

5 Leidy NK, Haase JE. Functional performance in people with chronic obstructive pulmonary disease: a qualitative analysis. ANS Adv Nurs Sci 1996;18:77-8.

6 Scioscia G, Blanco I, Arismendi E, et al. Different dysponea perception in COPD patients with frequent and infrequent exacerbations. Thorax 2017;72:117-21.

7 Wilkinson TM, Donaldson GC, Hurst JR, et al. Early therapy improves outcomes of exacerbations of chronic obstructive pulmonary disease. Am I Respir Crit Care Med 2004;169:1298-303. 